had a final infarct volume of $49.6 \mathrm{~cm}^{2}( \pm 46.3)$ versus a volume of $16.7 \mathrm{~cm}^{2}( \pm 13.8)$ for non-dominant occlusions $(\mathrm{p}=0.009)$. In patients with $>1 / 3^{\text {rd }} \mathrm{MCA}$ involvement on TTP images the infarct volume was $51.5 \mathrm{~cm}^{2}( \pm 47.6)$ versus an infarct volume of $28.1 \mathrm{~cm}^{2}( \pm 31.5)$ in patients with $\leq 1 / 3^{\text {rd }}$ MCA abnormality $(\mathrm{p}=0.03)$. There were $1,135,030$ AIS discharges nationally for 2013 for the same ICD-9 codes. A 4\% rate of M2 occlusions yields 45,401 potential patients with an M2 occlusion of which 38,137 can have a dominant branch involvement and hence the risk of significant ischemic injury.

Conclusion Patients with a dominant branch occlusion, whether superior or inferior, had larger TTP abnormalities $\left(>1 / 3^{\text {rd }} \mathrm{MCA}\right)$ and final infarct volumes. An estimated 37,137 AIS patients can have a dominant M2 occlusion with significant risk of ischemic injury. A functional as opposed to pure anatomical classification may allow selecting these patients for endovascular therapy.

Disclosures A. Rai: 1; C; Stryker Neurovascular. 2; C; Stryker Neurovascular. A. Tarabishy: None. P. Link: None. S. Boo: None. N. Lucke-Wold: None. J. Domico: None. J. Carpenter: None.

\section{0-009 ASPECTS SCORES AND DWI VOLUME: HOW WELL DO THEY CORRELATE?}

${ }^{1} \mathrm{~F}$ Hui, ${ }^{2} \mathrm{~J}$ Bullen, ${ }^{3} \mathrm{~S}$ John, ${ }^{3} \mathrm{G}$ Toth, ${ }^{3} \mathrm{~S}$ Hussain. ${ }^{1}$ Radiology, Johns Hopkins, Baltimore, MD; ${ }^{2}$ Quantitative Health Services, Cleveland Clinic, Cleveland, $\mathrm{OH} ;{ }^{3}$ Neurological Institute, Cleveland Clinic, Cleveland, $\mathrm{OH}$

\subsection{6/neurintsurg-2016-012589.9}

Recent trials for the management of large vessel occlusion for acute ischemic stroke have demonstrated better outcomes for intervention over IV tPA alone. Ideal imaging triage remains uncertain, however CT only paradigms, volumetric paradigms, penumbral paradigms and collateral paradigms have been proposed and used. The volumetric exclusion criteria employed in EXTEND-IA and SWIFT-PRIME may have contributed to mRS $0-2$ rates of $71 \%$ and $60 \%$ respectively. One of the appeals of MR estimation of irreversible "core" infarct is that it is the most accurate readily available modality. CT ASPECTS is more widely available, but may underestimate the volume of core.

Statistical methods The Pearson correlation coefficient was used to assess the amount of linear correlation between ASPECTS and DWI volume. The DWI volume values observed within each ASPECT score were then summarized. An empirical receiver operating characteristic (ROC) curve was used to summarize the accuracy of using ASPECTS to predict DWI volume less than $70 \mathrm{cc}$. The operating point furthest from the chance diagonal was selected as the optimal ASPECTS threshold and 95\% AgrestiCoull confidence intervals were calculated for the sensitivity and specificity at this cut point.

Results DWI ASPECTS and DWI volume had a strong negative correlation ( $\mathrm{r}=-0.76$; 95\% CI: $-0.67,-0.82)$, though there was a fair amount of variability in DWI volume within a given DWI ASPECT score. CT ASPECTS and DWI volume had a moderate negative correlation $(\mathrm{r}=-0.50 ; 95 \% \mathrm{CI}$ : $-0.36,-0.62)$, though there was considerable variability in DWI volume for a given CT ASPECT score. In this sample, there were 105 patients with DWI volume $<70 \mathrm{cc}$ and 27 with volume $\geq 70 \mathrm{cc}$. The area under the ROC curve for predicting DWI volume $<70 \mathrm{cc}$ was 0.93 for DWI ASPECTS and 0.81 for CT ASPECTS. The ASPECTS thresholds which maximized the overall rate of correct classification were 5 and 8 for DWI and CT, respectively. When DWI ASPECTS > 5 was considered positive for DWI volume $<70$, the estimated sensitivity and specificity were 0.88 (92/105; 95\% CI: 0.80 , $0.93)$ and $0.85(23 / 27 ; 95 \%$ CI: $0.67,0.95)$, respectively. When CT ASPECTS $>8$ was considered positive for DWI volume $<70$, the estimated sensitivity and specificity were 0.64 (67/105; 95\% CI: $0.54,0.72)$ and 0.85 (23/27; 95\% CI: $0.67,0.95)$, respectively.

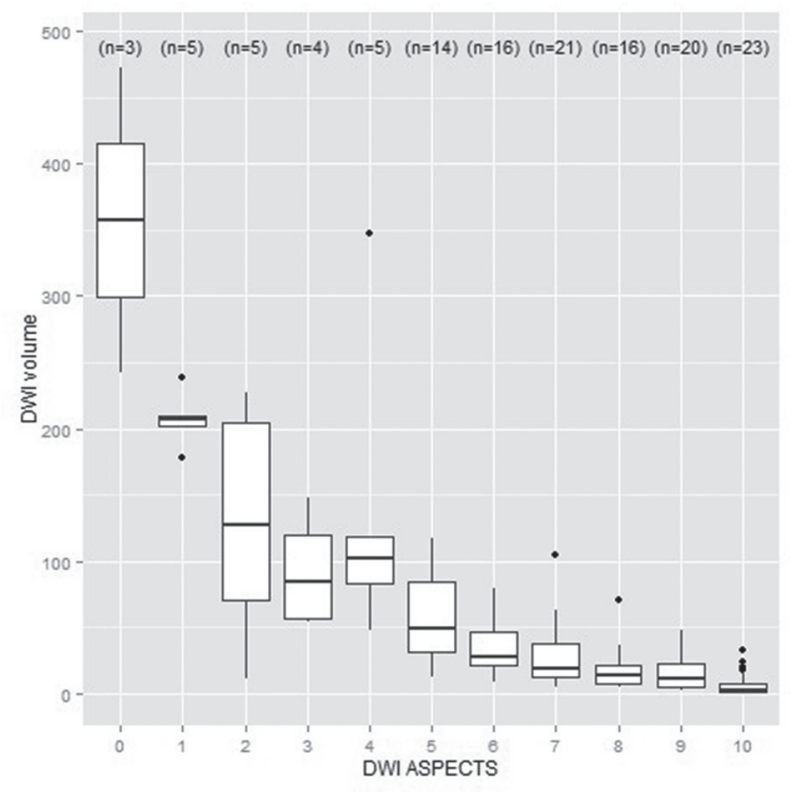

Abstract 0-009 Figure 1

Disclosures F. Hui: None. J. Bullen: None. S. John: None. G. Toth: None. S. Hussain: None.

\section{0-010 PLANNING FOR EFFICIENCY: SURVEY OF TECHNICAL AND WORKFLOW PRACTICES BEFORE MECHANICAL THROMBECTOMY}

${ }^{2} \mathrm{~A}$ Kansagra, ${ }^{1} \mathrm{G}$ Meyers, ${ }^{2} \mathrm{M}$ Kruzich, ${ }^{1} \mathrm{D}$ Cross, ${ }^{1} \mathrm{C}$ Moran, ${ }^{3} \mathrm{C}$ Derdeyn. ${ }^{1}$ Washington University School of Medicine, Saint Louis, MO; ${ }^{2}$ Barnes-Jewish Hospital, Saint Louis, MO; ${ }^{3}$ University of lowa Hospitals and Clinics, lowa City, IA

\subsection{6/neurintsurg-2016-012589.10}

Background Mechanical thrombectomy plays a critical role in the management of acute ischemic stroke due to emergent large vessel occlusion (ELVO). As healthcare systems adapt to more efficiently deliver patients with ELVO for timely thrombectomy, hospitals may benefit from increased awareness of successful workflows in place at other centers experienced with endovascular intervention.

Methods E-mail and phone interviews were conducted with endovascular team members at each of 30 high volume stroke centers. Each of these centers was certified as an Advanced Comprehensive or Advanced Primary Stroke Center. Questions were categorized into four major workflow steps of triage, team activation, transport, and case preparation.

Results During the triage workflow step, 53\% of surveyed institutions designate specific non-physician staff to respond to 
stroke alerts alongside physicians and facilitate timely triage. Imaging triage involves CT angiography and CT perfusion at $57 \%$ of institutions, CT angiography without CT perfusion at $30 \%$, and some use of MRI at $10 \%$. During the team activation step, the neurointerventionalist was contacted prior to completion of non-invasive imaging at $86 \%$ of institutions, and thus before triage was a decision to treat could be made. Likewise, the remaining neurointerventional team members were called in prior to making a decision to treat at 59\% of institutions. Team members were contacted directly by the neurointerventionalist at $63 \%$ of institutions, and by a hospital operator at $23 \%$. Once activated, the mean required arrival time for nurse and technologist staff was 30 minutes. During the transport workflow step, patients were permitted to be transported to the neurointerventional suite before team arrival at $43 \%$ of institutions. Emergency department staff were involved in transport at $87 \%$ of facilities, while the neurointerventional team was involved at only $20 \%$. For the case preparation step, procedural trays were set up in advance of team arrival at only $13 \%$ of institutions. Thrombectomy devices were stored in a centralized, easy-to-find location at 54\% of centers. A power injector for angiographic runs was consistently used at $43 \%$, but no institution left the injector loaded in anticipation of cases. Anesthesiology routinely participated in thrombectomies at $67 \%$ of institutions, though general anesthesia was only used consistently in only $21 \%$.

Conclusion Workflow processes related to triage, team activation, transport, and case preparation prior to mechanical thrombectomy vary widely between institutions. These differences may reflect institution-specific factors or incomplete awareness of best practices. Broader dissemination of best practices and successful workflows may allow institutions to develop more efficient systems of care than would otherwise be possible.

Disclosures A. Kansagra: None. G. Meyers: None. M. Kruzich: None. D. Cross: None. C. Moran: 2; C; Medtronic Neurovascular. 3; C; Medtronic Neurovascular. C. Derdeyn: None.

\section{0-011 SYMPTOMATIC INTRACRANIAL HEMORRHAGE AFTER REPERFUSION THERAPY - IMPACT OF DEFINITION ON ITS FREQUENCY}

${ }^{1} \mathbf{R}$ von Kummer, ${ }^{2} \mathrm{D}$ Frei, ${ }^{3} \mathrm{~A}$ Yoo, ${ }^{4} \mathrm{O}$ Zaidat, ${ }^{5} \mathrm{P}$ Khatri, ${ }^{6} \mathrm{R}$ Gupta, ${ }^{7} \mathrm{D}$ Lopes, ${ }^{8} \mathrm{H}$ Shownkeen, ${ }^{9} \mathrm{D}$ Meyer, ${ }^{9} \mathrm{H}$ Buell, ${ }^{9} \mathrm{~V}$ Bach, ${ }^{9} \mathrm{~S}$ Kuo, ${ }^{9} \mathrm{~A}$ Bose, ${ }^{9} \mathrm{~S}$ Sit, ${ }^{10} \mathrm{~J}$ Mocco. ${ }^{1}$ Universitätsklinikum Carl Gustav Carus, Dresden, Germany; ${ }^{2}$ Swedish Medical Center, Englewood, CO; ${ }^{3}$ Texas Stroke Institute, Plano, TX; ${ }^{4}$ St Vincent Mercy Hospital, Toledo, OH; ${ }^{5}$ University of Cincinnati, Cincinnati, OH; ${ }^{6}$ WellStar Health System, Marietta, GA; ${ }^{7}$ Rush University Medical Center, Chicago, IL; ${ }^{8}$ Central DuPage Hospital, Winfield, IL; ${ }^{9}$ Penumbra, Inc., Alameda, CA; ${ }^{10}$ Mount Sinai Health System, New York, NY

\subsection{6/neurintsurg-2016-012589.11}

Introduction There is currently no standardized criterion for a clinically defined symptomatic intracranial hemorrhages (sICH), particularly secondary to endovascular therapy of ischemic stroke. Recent randomized controlled trials evaluating the efficacy and safety of intra-arterial therapy have published results with varying complication rates based upon the sICH definition employed. To further elucidate the variability in sICH outcome, the present study subjects the THERAPY cohort to definitions of sICH from similar trials.

Materials and methods The multicenter THERAPY trial enrolled a total of 108 patients randomized to either IV-tPA monotherapy or a combined thrombolytic and intra-arterial therapy. Angiography was performed at presentation and at 24 hours from intervention. To meet the present study criterion, patients included in the study must have the relevant data document for evaluation of sICH presence, defined in the THERAPY trial as $24 \mathrm{~h} \mathrm{CT} \mathrm{evidence}$ of an ECASS-defined intracranial hemorrhage and a worsening of $\geq 4$ on the National Institute of Health Stroke Scale and compared to outcomes when subjecting the same cohort to definitions from related trials.

Results In total, 105 patients from the THERAPY trial met the criteria for analysis. Under the study definition (Definition A), the rate of sICH was observed to be $9.5 \%$ (10/105) for both arms of the THERAPY trial, with 9.3\% (4/43) of patients documented for the combined therapy arm. More narrow definitions for sICH, such as those employed by

Abstract 0-011 Table 1

\begin{tabular}{|c|c|c|c|c|c|c|c|c|}
\hline \multirow[t]{2}{*}{$\begin{array}{c}\text { Stroke } \\
\text { Trials }\end{array}$} & \multicolumn{2}{|c|}{$\begin{array}{c}\text { Definition A: } \\
24 \mathrm{hr} \text { CT evidence of an } \\
\text { ECASS defined ICH and a } \\
\text { worsening in NIHSS score of } \\
\geq 4 \text { from baseline }\end{array}$} & \multicolumn{2}{|c|}{$\begin{array}{l}\text { Definition B: } \\
\text { Any PH1, PH2, RIH, SAH, or } \\
\text { IVH associated with a } \geq 4 \\
\text { points worsening on the } \\
\text { NIHSS within } 24 \mathrm{hr}\end{array}$} & \multicolumn{2}{|c|}{$\begin{array}{c}\text { Definition C: } \\
\text { PH2 within } 36 \mathrm{hr} \text { of } \\
\text { treatment combined with } \geq 4 \\
\text { point increase in NIHSS from } \\
\text { baseline, or the lowest } \\
\text { NIHSS value between } \\
\text { baseline and } 24 \mathrm{hr}\end{array}$} & \multicolumn{2}{|c|}{$\begin{array}{c}\text { Definition D: } \\
\text { New intracranial } \\
\text { hemorrhage (ICH, SAH, IVH, } \\
\text { SDH) associated with clinica } \\
\text { evidence of neurological } \\
\text { worsening associated with } \\
\text { NIHSS score of } \geq 2 \text { points } \\
\text { different from baseline }\end{array}$} \\
\hline & Control & Intervention & Control & Intervention & Control & Intervention & Control & Intervention \\
\hline ESCAPE & - & - & - & - & - & - & $2.7 \%$ & $3.6 \%$ \\
\hline EXTEND IA & - & - & - & - & $6 \%$ & $0 \%$ & - & - \\
\hline REVASCAT & $1.9 \%$ & $4.9 \%$ & - & - & $1.9 \%$ & $1.9 \%$ & - & - \\
\hline $\begin{array}{l}\text { SWIFT } \\
\text { PRIME }\end{array}$ & - & - & $3.1 \%$ & $0.0 \%$ & - & - & - & - \\
\hline MR CLEAN & $6.4 \%$ & $7.7 \%$ & - & - & - & - & - & - \\
\hline THERAPY & $9.7 \%$ & $9.3 \%$ & $4.8 \%$ & $2.3 \%$ & $4.8 \%$ & $0.0 \%$ & *N/A & $* N / A$ \\
\hline
\end{tabular}

* Adjudication in progress

Note: $\mathrm{ICH}=$ Intracranial Hemorrhage, $\mathrm{PH}=$ Parenchymal Hemorrhage, RIH = Remote Intracranial Hemorrhage, SAH = Subarachnoid

Hemorrhage, IVH = Intraventricular Hemorrhage, SDH = Subdural Hemorrhage 